\title{
The Late Demonstration of the Parry-Romberg Syndrome
}

\author{
Persechino $\mathrm{S}^{\mathbf{1}}$, Di Vito $\mathrm{E}^{\mathbf{1}}$, Lupi $\mathrm{F}^{\mathbf{1}}$, Romano ${ }^{1}{ }^{1}$, Parisella $\mathrm{FR}^{3}$, Persechino $\mathrm{F}^{\mathbf{2}}$, Mallel $\mathrm{G}^{4}$, Tammaro $\mathrm{A}^{\mathbf{1}}$ \\ ${ }^{1}$ NESMOS Department, Dermatology Unit, Sant'Andrea Hospital, Faculty of Medicine and Psychology, University of Rome "Sapienza" \\ ${ }^{2}$ Dermatology Unit, University of Modena, Italy. \\ ${ }^{3}$ Faculty of Medicine, Towson University, Towson City, Maryland (USA) \\ ${ }^{4}$ Clinical and Molecular Medicine's Department, Sant'Andrea Hospital, Faculty of Medicine and Psychology, University of Rome "Sapienza"
}

Received: April 04, 2016; Accepted: April 20, 2016; Published: May 09, 2016

*Corresponding author: Severino Persechino, PhD, NESMOS Department, Dermatology Unit, Sant'Andrea Hospital, Faculty of Medicine and Psychology, Via di Grottarossa, 1035, 00189 Rome, Italy, Tel: +39-06-33775269; Fax: +39-06-33775378; E-mail: severino.persechino@uniroma1.it

\begin{abstract}
We report the case of a 46 years-old woman, who developed a linear lesion sclero-atrophic from the medial frontal region and which expanded in cranio-caudal sense, with subsequent skin and dermic atrophy.
\end{abstract}

A biopsy was performed and confirmed a dermal-epidermal sclerosis. These data, associated with the clinical characteristics, let us to diagnose a Parry-Romberg Syndrome.

Parry Romberg syndrome is a scleroatrophic syndrome, linear of the face which presents a localized partial hemiatrophy without systemic involvement.

In addition, the patient reported diffuse pain in the joints, previous autoimmune thyroiditis and reduced salivation and lacrimation bilaterally with the positive Schirmer test. The data confirmed the hypothesis of a possible S. Sjogren associated with Parry-Romberg.

This medical case is suggestive because the Parry-Romberg is a rare syndrome in adults, and usually not observed in other autoimmune disease, as S. Sjogren.

Keywords: Parry-Romberg Syndrome; Skin atrophy; Coupe de sabre; Sjogren Syndrome

\section{Introduction}

Parry-Romberg syndrome, also known as progressive hemifacial atrophy, is a rare neurocutaneous syndrome, characterized by unilateral facial atrophy affecting the skin, subcutaneous tissue, muscles, and sometimes extending to the osteocartilaginous structures. Although the etiopathogenesis is still unknown, an autoimmune mechanism is suspected and the syndrome may be a variant of localized scleroderma. The syndrome has a higher prevalence in females and typically appears between $5-15$ years of age $[1,2]$.

The clinical manifestations are highly variable. Initially, the disease involves the region of the buccinator and temporal muscles, and gradually extends to the whole unilateral facial side. It causes atrophy of skin and adnexa, deepening to the subcutaneous tissue. The mouth and nose are typically deviated towards the affected side of the face.

Initially, it begins as an area of sclerosis from the frontal region of the scalp, with hair loss. Subsequently, it appears a scar which extends along the median part of the face, called "coup de sabre" [7].

In $20 \%$ of cases, the affected area may be predisposed to altered pigmentation $[4,5]$.

Ocular involvement is relatively rare. [3]

Also, Parry-Romberg Syndrome is usually not observed in other autoimmune disease, including Sjogren Syndrome, which is a chronic inflammatory autoimmune disease characterized by destruction of exocrine glands, in particular lacrimal and salivary glands $[3,6,8]$.

\section{Materials and Methods}

We report the case of 46 years-old woman who, during 3 months, developed a linear sclero-atrophic lesion in the medial frontal region, extending in cranio-caudal sense, with involvement of the glabellar region, nasolabial fold and ipsilateral chin angle, with subsequent skin and dermic atrophy (Fig. I-II).

We performed a cutaneous biopsy (Fig. III), which highlighted sclerosis of the dermal-epidermal junction and the reticular dermis in the context of which it was present a lymphomononucleated chronic inflammation with perivascular and periadnexal distribution. In sections, at the level of the hair follicle hairy, three structures were visible in complete atrophy.

According to the clinical and histological characteristics, it is done diagnosis of a saber scleroderma (S. Parry-Romberg).

The patient has performed the Magnetic Resonance Imaging (MRI) and electromyography, but in our case it did not highlight alterations worthy of note.

In addition, the patient reported diffuse pain in the joints, previous autoimmune thyroiditis and symptoms and signs suggestive of Sjogren Syndrome, as bilateral xerophthalmia, xerostomia with the positive Schirmer test. 


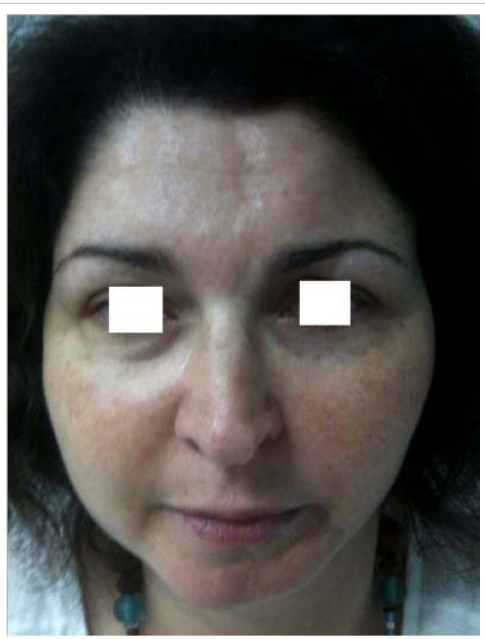

Figure I: Patient with a linear slero-atrophic lesion in the medial frontal region, with involvement of forehead, nose and chin (coup de sabre).

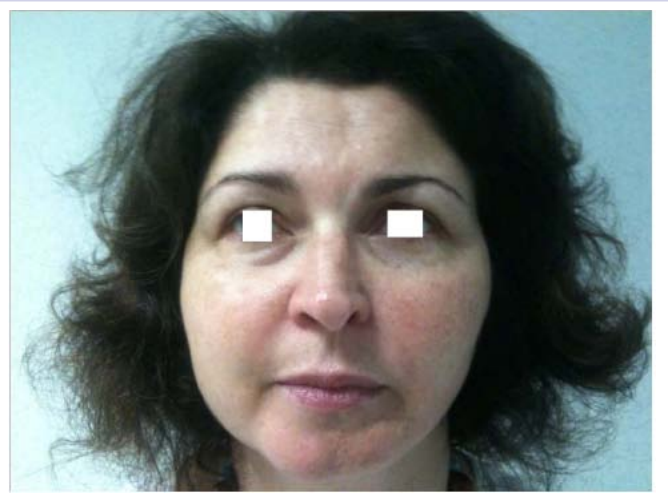

Figure II: Patient with a linear slero-atrophic lesion in the medial frontal region, with involvement of forehead, nose and chin (coup de sabre).

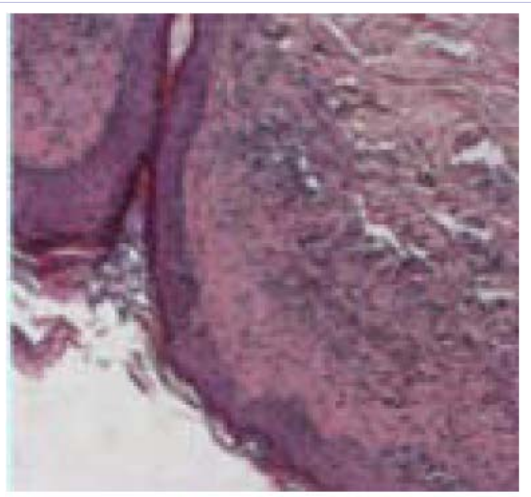

Figure III: Interstitial lichenoid chronic infiltration forming a band in the reticular dermis surrounding a pilar follicle. H\&E 100X. in a 46-years-old woman [9].

In addition, an other particular aspect is that Parry-Romberg Syndrome is usually not observed in other autoimmune disease, while in our case it is associated with Sjogren Syndrome. In fact the association between Parry-Romberg and the $S$. Sjogrenreopens the discussion about autoimmunity because, in our case, the patient is suffering simultaneously from a localized connectivitis, as Parry-Romberg, and a systemic connectivitis, as Sjogren Syndrome [7].

\section{References}

1. Asai M, Sago T, Utani A. Parry-Romberg syndrome associated with contralateral cerebral atrophy.Eur J Dermatol. 2015;25(6):624-625. doi: $10.1684 /$ ejd.2015.2651.

2. ydın H, Yologlu Z, Sargın H, Metin MR. Parry-Romberg syndrome. Physical, clinical, and imaging features

3. Neurosciences (Riyadh). 2015;20(4):368-371. doi: 10.17712/ nsj.2015.4.20150142.

4. Fea AM, Aragno V, Briamonte C, Franzone M, Putignano D, Grignolo FM. Parry Romberg syndrome with a wide range of ocular manifestations: a case repot. BMC Ophthalmol. 2015;15:119. doi: 10.1186/s12886015-0093-0.

5. Wong M, Phillips CD, Hagiwara M, Shatzkes DR. Parry Romberg Syndrome: 7 Cases and Literature Review.

6. AJNR Am J Neuroradiol. 2015;36(7):1355-1361. doi: 10.3174/ajnr. A4297.

7. Doolittle DA, Lehman VT, Schwartz KM, Wong-Kisiel LC, Lehman JS, Tollefson MM. CNS imaging findings associated with Parry-Romberg syndrome and en coup de sabre: correlation to dermatologic and neurologic abnormalities.

8. Neuroradiology. 2015;57(1):21-34. doi: 10.1007/s00234-014-14486.

9. Nair PA, Patel NH, Diwan NG. Parry-romberg syndrome: a linear variant of scleroderma with discoid lupus erythematosus on scalp an association. Int J Trichology. 2014;6(2):71-74. doi: 10.4103/09747753.138594 .

10. Khan M, Khan M, Negi R, Gupta N. Parry Romberg syndrome with localized scleroderma: A case report.

11. Clin Exp Dent. 2014;6(3):e313-316. doi: 10.4317/jced.51409. eCollection 2014 Jul.

12. Walker RH, Fink JK. Morphea and Parry-Romberg syndrome associated with a mixed movement disorder.

13. Parkinsonism Relat Disord. 2013;19(12):116911-116970. doi: 10.1016/j.parkreldis.2013.07.025.

14. Jun JH, Kim HY, Jung HJ, Lee WJ, Lee SJ, Kim do, W et al. Parry-romberg syndrome with en coup de sabre.

15. Ann Dermatol. 2011;23(3):342-347. doi: 10.5021/ad.2011.23.3.342.

Laboratory examinations showed negative antibody.

\section{Conclusion}

This medical case is suggestive because the Parry-Romberg is a rare syndrome in adults, in fact it typically appears between $5-15$ years of age, while in our case the disease was diagnosed 